\title{
Distribution kinetics theory of Ostwald ripening
}

\author{
Giridhar Madras* \\ Department of Chemical Engineering, Indian Institute of Science, Bangalore 560 012, India
}

Benjamin J. McCoy

Department of Chemical Engineering and Materials Science, University of California, Davis, California 95616

\begin{abstract}
Ostwald ripening occurs near equilibrium conditions when larger clusters grow at the expense of dissolving smaller clusters. We propose that ripening kinetics for growth and dissolution can be represented by a general population balance equation (PBE) for the cluster size distribution (CSD). This PBE can also describe cluster growth or dissolution in the absence of ripening. The Kelvin equation provides the effect of interfacial energy on solubility in terms of the cluster radius. The continuity equation conventionally applied to ripening or cluster growth is obtained as a Taylor series expansion of the governing PBE. Numerical and moment solutions of the PBE show the evolution of the CSD. The cluster number density declines, and the average cluster mass increases. The variance can initially increase as the CSD broadens by growth of large clusters, and then decrease until eventually vanishing. The final state after a long time is a single large cluster in equilibrium with the fluid solution.
\end{abstract}

\section{INTRODUCTION}

Ostwald ripening is the final stage of a first-order phase transition for condensation of a metastable phase. ${ }^{1}$ The first stage is nucleation, either homogeneous (a free energy barrier is surmounted to form critical-sized clusters) or heterogeneous (nucleation sites present in the system allow molecular, i.e., monomer, deposition). The second stage is cluster growth by monomer deposition, which depletes the metastable phase of monomer and causes stable clusters to grow regardless of their size. Due to the random deposition of monomers from the metastable phase, this growth process yields a size distribution of clusters, even when the criticalsized clusters formed by homogeneous nucleation have the same uniform mass. This was recently illustrated for vaporliquid nucleation and growth ${ }^{2}$ and crystal growth or dissolution. ${ }^{3}$ The free energy of the clusters is likewise distributed, due to the effect of surface curvature of different sized clusters on interfacial energy. ${ }^{4}$ The curvature, and hence the interfacial free energy, is reduced by increasing the cluster size. Smaller clusters thus are driven to dissolve and give up their monomers to larger clusters: This final stage of the phase transition is Ostwald ripening or coarsening, also called isothermal recrystallization ${ }^{5}$ when the phase transition is liquid to solid. In this paper, cluster is a generic term for the condensed phase, whether it is a liquid droplet or solid precipitate.

Ripening models are based on the Kelvin (or GibbsThomson) equation, ${ }^{6-9}$ which gives the relationship for the ratio of interfacial energy to thermal energy such that smaller *Author to whom correspondence should be addressed. Telephone:
91-080-309-2321. Fax: 91-080-360-0683. Electronic mail: giridhar@chemeng.iisc.ernet.in clusters are less stable than larger ones and therefore larger clusters grow at the expense of smaller ones. The Kelvin equation ${ }^{6}$ for a given supersaturation provides an expression for the critical nucleus size, above which the cluster grows, below which the cluster is unstable and dissolves unless fluctuations allow homogeneous nucleation. Clusters become more soluble as they become smaller, and eventually disappear, yielding up their mass for growth of larger clusters. ${ }^{10}$ The ultimate state is reached when but one cluster remains in equilibrium with the monomer solution. Although most papers on the theory of ripening mention the reduction in cluster numbers during ripening, this effect is sometimes neglected altogether. Obviously, the final equilibrium condition of one large cluster cannot be attained unless denucleation is considered. Some models focus instead on the mass transfer rate for dissolution and growth. Conventional theories are usually based on first-order differential equations for cluster growth of the cluster size distribution. ${ }^{1,6,7}$ The growth rate is represented by the difference between the solution concentration and its equilibrium saturation value as the driving force. When supersaturation is much greater than unity, homogeneous nucleation can occur, and all stable clusters (larger than critical nucleus size) will grow. The goal is to describe ripening for a distribution of clusters when supersaturation causes larger clusters to grow while smaller clusters vanish (denucleate). Some ripening models apply to only a few particles, ${ }^{11}$ rather than the more interesting distribution of particle sizes. Typically the exponential in the Kelvin equation is approximated by the first-order linear term. ${ }^{6,7}$ An analytical asymptotic solution is usually sought that applies for long time and is independent of initial conditions. ${ }^{6}$ Most theories do not explicitly represent the evolution to a single large cluster, which would be expressed as a Dirac $\delta$ distribution. 
Lifshitz and Slyozov (LS) ${ }^{12}$ and Wagner (W) ${ }^{13}$ were among the first to study the cluster mass distribution, but assumed the monomer concentration is constant at its equilibrium value. Marqusee and Ross ${ }^{1}$ showed that the LSW solution can be represented as leading terms in an expansion of the long time solution. $\mathrm{Venzl}^{8}$ solved the governing firstorder nonlinear differential equation numerically, and assumed that clusters vanished at a rate varying exponentially or inversely with time. Bhakta and Ruckenstein ${ }^{7}$ more recently based a stochastic theory of ripening on a discrete microscopic continuity equation that generalized the LSW differential equation with rate constants assumed independent of cluster size.

The present objective is to formulate a new approach to Ostwald ripening (or isothermal recrystallization) that accounts for the evolution of the cluster size distribution (CSD) expressed in terms of the cluster mass, rather than its radius. The distribution-kinetics approach with single monomer addition and dissociation is reversible and is generally applicable to growth, dissolution, or ripening phenomena. Based on a rigorous mass balance, nucleation (or denucleation) appears as a source (or sink) term representing the nucleation rate. $^{6}$ Denucleation of unstable clusters ensures that the cluster number decreases as required for a realistic model of ripening. The reversibility ensures that a closed system relaxes to equilibrium, where the system is saturated. The equilibrium is dynamic, with monomer addition and dissociation continuing at equilibrium. For a continuous distributionkinetics theory, the second moment can be shown to grow during ripening, but because of denucleation, the variance decreases. The CSD spreads and large particles grow larger. Smaller clusters shrink, releasing their mass to the solution. Eventually, after a long time, only one large particle would remain, which clearly requires an explanation based on a discrete distribution model. The continuous distribution kinetics theory applies only for a very large number of clusters, so that as the number of clusters declines, a discrete model must eventually be implemented. A numerical solution for discrete PBE shows ripening that continues until cluster mass is accumulated into one large cluster, subject to conservation of the mass initially available. The discussion applies for various expressions for rate coefficients.

The present paper is organized as follows: We first present our approach based on distribution kinetics, and formulate a population balance for the reversible addition of monomer to a cluster. Next the evolution to an equilibrium state is described. In Sec. IV we develop the governing equations for ripening, and in Sec. $\mathrm{V}$ we discuss the numerical solution to these equations. Finally, we present and discuss the results of the calculations, and draw conclusions.

\section{DISTRIBUTION KINETICS}

The CSD is defined by $c(x, t) d x$, which represents the concentration of clusters at time $t$ in the mass range $(x, x$ $+d x)$. Moments are defined as integrals over the mass,

$$
c^{(n)}(t)=\int_{o}^{\infty} c(x, t) x^{n} d x .
$$

The zeroth moment $c^{(0)}(t)$ is the molar (or number) concentration of clusters, and the first moment $c^{(1)}(t)$ is the timedependent cluster mass concentration (mass/volume). The ratio of the two is the average cluster mass, $c^{\text {avg }}=c^{(1)} / c^{(0)}$. The variance $c^{\mathrm{var}}=c^{(2)} / c^{(0)}-\left[c^{\mathrm{avg}}\right]^{2}$ and the polydispersity, $c^{(2)} c^{(0)} / c^{(1) 2}$, are measures of the CSD broadness. The molar concentration, $m(t)$, of solute monomer of molecular weight $x_{m}$ is the zeroth moment of the monomer distribution, $m(x, t)=m(t) \delta\left(x-x_{m}\right)$.

The deposition or condensation process by which monomers of mass $x^{\prime}=x_{m}$ are reversibly added to or dissociated from a cluster of mass $x$ can be written as a reactionlike process

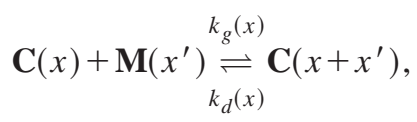

where $\mathbf{C}(x)$ represents the cluster of mass $x$ and $\mathbf{M}\left(x^{\prime}\right.$ $\left.=x_{m}\right)$ is the monomer. This process intrinsically conserves mass, and is most naturally represented by balance equations in terms of mass $x$ rather than cluster radius $r$. The balance equations governing the cluster distribution, $c(x, t)$, and the monomer distribution, $m(x, t)=m(t) \delta\left(x-x_{m}\right)$, are thus based on mass conservation: ${ }^{2}$

$$
\begin{aligned}
\partial c(x) / \partial t= & -k_{g}(x) c(x) \int_{o}^{\infty} m\left(x^{\prime}\right) d x^{\prime}+\int_{o}^{x} k_{g}\left(x-x^{\prime}\right) \\
& \times c\left(x-x^{\prime}\right) m\left(x^{\prime}\right) d x^{\prime}-k_{d}(x) c(x)+\int_{x}^{\infty} k_{d}\left(x^{\prime}\right) \\
& \times c\left(x^{\prime}\right) \delta\left(x-\left(x^{\prime}-x_{m}\right)\right) d x^{\prime}-I \delta\left(x-x^{*}\right)
\end{aligned}
$$

and

$$
\begin{aligned}
\partial m(x) / \partial t= & -m(x) \int_{o}^{\infty} k_{g}\left(x^{\prime}\right) c\left(x^{\prime}\right) d x^{\prime}+\int_{x}^{\infty} k_{d}\left(x^{\prime}\right) \\
& \times c\left(x^{\prime}\right) \delta\left(x-x_{m}\right) d x^{\prime}+I \delta\left(x-x^{*}\right) x^{*} / x_{m} .
\end{aligned}
$$

According to the molecularity of Eq. (2.2), addition reactions are second-order in $c(x, t)$ and $m(x, t)$, whereas dissociation reactions are first-order in $c(x, t)$. Nucleation of clusters of mass $x^{*}$ at rate $I$ are source terms or, in this case, sink terms for denucleation. Initial conditions are $c(x, t=0)=c_{0}(x)$ and $m(x, t=0)=m_{o} \delta\left(x-x_{m}\right)$. The growth and dissociation rate coefficients, $k_{g}$ and $k_{d}$, may in general depend upon $x$ and local thermodynamic conditions, and must therefore be expressed as constitutive relations. Ripening means that all clusters are either growing or dissolving at rates depending on their size as determined by the Kelvin equation. Powerlaw expressions allow for a kinetic dependence on cluster size, and thus we consider $k_{g}(x)=\gamma x^{\lambda}$ and $k_{d}(x)=\kappa x^{\nu}$, where $\gamma$ and $\kappa$ in general are functions of temperature. The coefficients $\gamma$ and $\kappa$ may depend on the deposition or dissociation processes or on the mode of transport to and from the 
cluster surface. When the constitutive relations are substituted and the moment operation, defined by Eq. (2.1), is applied, the general moment equations are

$$
\begin{aligned}
d c^{(n)} / d t= & \gamma m\left[-c^{(\lambda+n)}+\sum_{j=0}^{n}\left(\begin{array}{l}
n \\
j
\end{array}\right) x_{m}^{j} c^{(\lambda+n-j)}\right] \\
& +\kappa\left[-c^{(\nu+n)}+\sum_{j=0}^{n}\left(\begin{array}{l}
m \\
j
\end{array}\right) x_{m}^{j}(-1)^{j} c^{(\nu+n-1)}\right]-i x^{* n}
\end{aligned}
$$

and

$$
d m / d t=-\gamma m c^{(\lambda)}+\kappa c^{(\nu)}+I x^{*} / x_{m} .
$$

When $\lambda=\nu$, the equations reduce to those proposed by McCoy. ${ }^{3}$ It follows from Eq. (2.5) that the cluster moment equations for $n=0,1$, and 2 are

$$
\begin{aligned}
d c^{(0)} / d t= & -I, \\
d c^{(1)} / d t= & x_{m} \gamma m c^{(\lambda)}-x_{m} \kappa c^{(\nu)}-I x^{*}, \\
d c^{(2)} / d t= & x_{m} \gamma m\left[2 c^{(\lambda+1)}+x_{m} c^{(\lambda)}\right] \\
& +\kappa x_{m}\left[-2 c^{(\nu+1)}+x_{m} c^{(\nu)}\right]-I x^{* 2} .
\end{aligned}
$$

Equations (2.6) and (2.8) demonstrate that mass is then conserved for any values of $\lambda$ and $\nu$ thus

$$
x_{m} d m / d t=-d c^{(1)} / d t .
$$

For the initial conditions, $m(t=0)=m_{o}^{(0)}$ and $c^{(1)}(t=0)$ $=c_{o}^{(1)}$, integration of Eq. (2.10) gives

$$
x_{m}\left[m_{o}^{(0)}-m(t)\right]-c_{o}^{(1)}+c^{(1)}(t),
$$

so that the decrease in solute mass is balanced by an increase in cluster mass.

\section{EVOLUTION TO EQUILIBRIUM}

The evolution to equilibrium can be understood by formulating thermodynamic equilibrium conditions. We consider that the free energy of a cluster, $\mu$, relative to the monomer solution, depends upon its mass $x$ according to a polynomial in $x{ }^{14,15}$

$$
\mu(x)=\sum_{j=0}^{\infty} \mu_{j} x^{j},
$$

where the coefficients $\mu_{j}$ are independent of $x$ and $t$. The system total free energy (relative to the monomer solution) therefore is given by

$$
G(t)=\int_{o}^{\infty} \mu(x) c(x, t) d x=\sum_{j=0}^{\infty} \mu_{j} c^{(j)}(t) .
$$

Minimizing the free energy at equilibrium, $d G / d t=0$, therefore implies that derivatives of all moments must also vanish, $d c^{(j)} / d t=0, j \geqslant 0$. Equilibrium is thus established by a hierarchical sequence in which the moments vanish. From Eq. (2.7) when $d c^{(0)} / d t=0$, the number of clusters must be constant with $I=0$, when denucleation ceases. By Eq. (2.8) the first moment becomes constant, as well as the monomer concentration, $d m / d t=0$. Either Eq. (2.6) or (2.8) yields the equilibrium solubility condition,

$$
m_{\mathrm{eq}}=\kappa c_{\mathrm{eq}}^{(\nu)} / \gamma c_{\mathrm{eq}}^{(\lambda)} .
$$

The temperature and physical property effects ${ }^{6}$ (interfacial energy, particle density, particle surface curvature) are expressed through the Kelvin equation, ${ }^{11}$

$$
m_{\mathrm{eq}}=m_{\infty} \exp (\Omega)
$$

with

$$
\Omega=2 \sigma \nu / r R T
$$

in terms of the gas constant $R$, monomer molar volume $\nu$ $=x_{m} / \rho$ in the cluster, excess surface free energy $\sigma$, radius of an assumed spherical cluster $r$, and equilibrium solute concentration for a plane cluster surface $m_{\infty} . \rho$ is the mass density of the crystals. The ratio of interfacial energy, $2 \sigma \nu / r$, to thermal energy, $R T$, determines the size effect on solubility. If a cluster has a shape other than spherical, a more involved expression is required to account for the effect of surface shape on free energy (for example, for a rectangular crystal $^{11}$ ). Equation (3.4) determines that larger clusters are more stable than smaller clusters, so that smaller clusters tend to dissolve while larger clusters grow by deposition of the dissolved monomer. Obviously the smaller clusters can dissolve totally, reducing the number of clusters. The smallest stable cluster has radius

$$
r^{*}=2 \sigma \nu /(R T \ln S)
$$

from which we have $x^{*}=(4 / 3) \pi \rho r^{* 3}$ as the critical cluster mass. By this reasoning, eventually only a single large cluster will exist, of a size consistent with the mass balance, Eq. (2.11),

$$
x_{m}\left[m_{o}^{(0)}-m_{\mathrm{eq}}^{(0)}\right]=c_{\mathrm{eq}}^{(1)}-c_{o}^{(1)} .
$$

As the number of clusters becomes small, this evolution to a single cluster must be expressed by a discrete population balance theory. The continuous model shows the second moment initially increasing [Eq. (2.9)] as larger particles get larger and smaller particles get smaller. To aid the solution of the PBE, Eqs. (3.3) and (3.4) provide an expression for $\kappa$ that introduces the thermodynamic effects,

$$
\kappa=\gamma m_{\infty} e^{\Omega} c_{\mathrm{eq}}^{(\lambda)} / c_{\mathrm{eq}}^{(\nu)} .
$$

Driven by the cluster interfacial energy, ripening occurs when $\lambda=\nu$, which serves as a realistic condition for evaluating the present model.

The molar concentration of solute, $m(t)$, even though near equilibrium, may change during ripening as the average cluster radius $r$ grows. Final equilibrium is reached when $m \rightarrow m_{\text {eq }}$ and all but one cluster has diminished to vanishing size, thus minimizing the free energy. The final cluster distribution is therefore the Dirac $\delta$ distribution for a single cluster, 


$$
c_{\mathrm{eq}}(x)=\delta\left(x-c_{\mathrm{eq}}^{\mathrm{avg}}\right) / N_{A},
$$

and therefore

$$
c_{\mathrm{eq}}^{(n)}=\left(c_{\mathrm{eq}}^{\mathrm{avg}}\right)^{n} / N_{A},
$$

where $N_{A}$ is Avogadro's number and $c_{\text {eq }}^{\text {avg }}$ is a large mass embodying the mass of dissolved clusters. According to Eq. (3.4), at long time when $d m / d t=0$ the supersaturation becomes constant and approaches $m_{\mathrm{eq}} / m_{\infty}=e^{\Omega_{\mathrm{eq}}}$, by definition. For the single large cluster at this final state, $\Omega_{\mathrm{eq}} \approx 0$, and therefore $m_{\mathrm{eq}} / m_{\infty}=S_{\mathrm{eq}} \approx 1$. The cluster radius $r$ at any time is found from the average cluster mass, $(4 \pi / 3) r^{3} \rho$ $=c^{\text {avg }}$, thus

$$
r=\left[3 c^{\mathrm{avg} /(4 \pi \rho)]^{1 / 3}}\right.
$$

and $c^{\mathrm{avg}} \rightarrow c_{\mathrm{eq}}^{\mathrm{avg}}$ at the long-time final state.

\section{EQUATIONS FOR RIPENING}

We consider ripening to occur when competing growth and dissolution processes for large and small clusters cause denucleation which decreases the number of clusters. The CSD changes according to Eq. (2.3), which becomes, when the integrations over the Dirac distributions are performed, ${ }^{2,16}$ the finite-difference differential equation,

$$
\begin{aligned}
\partial c(x, t) / \partial t= & -k_{g}(x) c(x) m+k_{g}\left(x-x_{m}\right) c\left(x-x_{m}\right) m \\
& -k_{d}(x) c(x)+k_{d}\left(x+x_{m}\right) c\left(x+x_{m}\right) \\
& -I \delta\left(x-x^{*}\right) .
\end{aligned}
$$

The discrete PBE thus can be considered a special case of the continuous PBE. The equation is similar to nearest-neighbor master equations used to solve many problems of kinetics and energetics. ${ }^{17}$ Equation (4.1) shows that $c(x, t)$ increases by addition of mass $x_{m}$ to the reactant of mass $\left(x-x_{m}\right)$ and decreases by the loss of reactant of mass $x$. The dissociation of mass $x_{m}$ from reactant of mass $\left(x+x_{m}\right)$ increases $c(x, t)$ while the loss of reactant of mass $x$ decreases $c(x, t)$. When $k_{g}$ and $k_{d}$ are constant (independent of $x$ ) and nucleation rate is zero, Eq. (4.1) is similar to the equation proposed for (reversible) polymerization and depolymerization kinetics by McCoy and Madras. ${ }^{16}$ Even when $k_{g}$ and $k_{d}$ are constant, however, there is no known analytical solution to Eq. (4.1) because of the presence of time-dependent $m(t)$ in Eq. (2.6) or (2.11). Fortunately, numerical solutions to such ordinary differential equations are straightforward.

The conversion of master equations such as Eq. (4.1) into partial differential Fokker-Planck equations is well known. ${ }^{17}$ If in Eq. (4.1) the rate coefficients and CSDs expressed as functions of $x-x_{m}$ or $x+x_{m}$ are expanded in their Taylor series, one obtains

$$
\begin{aligned}
\partial c(x) / \partial t= & -x_{m}\left\{\partial\left[k_{g}(x) c(x)\right] / \partial x-\left(x_{m} / 2\right) \partial^{2}\left[k_{g}(x) c(x)\right] / \partial x^{2}+\ldots\right\} \\
& +x_{m}\left\{\partial\left[k_{d}(x) c(x)\right] / \partial x+\left(x_{m} / 2\right) \partial^{2}\left[k_{d}(x) c(x)\right] / \partial x^{2}+\ldots\right\}-I \delta\left(x-x^{*}\right) .
\end{aligned}
$$

When third- and higher-order derivative terms are neglected, we have a convective-diffusion equation (with denucleation rate, $I)$ for $c(x, t)$. It is obvious, therefore, why the CSD must change both its average and variance. If secondderivative terms are neglected, one has the approximate continuity equation in $x$ space,

$$
\partial c(x) / \partial t+\partial[V(x) c(x)] / \partial x=-I \delta\left(x-x^{*}\right),
$$

where

$$
V(x)=x_{m} k_{g}(x)\left[m-k_{d}(x) / k_{g}(x)\right] .
$$

A similar equation with $x$ replaced by cluster radius $r$ (see the Appendix) is customarily applied in ripening models. ${ }^{1,8,12,13}$ The present theory is thus a generalization of the approximate models for Ostwald ripening based on Eq. (4.3) as the governing differential equation. Requiring Eq. (4.4) to satisfy the equilibrium condition, $k_{d}(x) / k_{g}(x)=m_{\text {eq }}=m_{\infty} \exp (\Omega)$, and allowing rate coefficients to be independent of $x$ gives

$$
V(x)=k_{g}\left[m-m_{\infty} \exp (\Omega)\right] .
$$

The growth rate coefficient $k_{g}$ may be specific to a stirred system, or may be related to the monomer diffusion coefficient in an unstirred system. Equation (4.5) allows the driv- ing force for ripening to vanish when equilibrium is attained. In Eq. (4.5), $V$ depends upon $x=(4 \pi / 3) r^{3} \rho$ through $\Omega$, which varies inversely with $r$ [Eq. (3.5)].

\section{NUMERICAL SOLUTION}

More general than the conventional continuity equation (4.3), the difference-differential equation, Eq. (4.1), can be solved by a finite difference method. When rate coefficients have power-law dependence on $x$, Eq. (4.1) becomes

$$
\begin{aligned}
\partial c(x, t) / \partial t= & -\gamma x^{\lambda} c(x) m+\gamma\left(x-x_{m}\right)^{\lambda} c\left(x-x_{m}\right) m \\
& -\kappa x^{\nu} c(x)+\kappa\left(x+x_{m}\right)^{\nu} c\left(x+x_{m}\right) \\
& -I \delta\left(x-x^{*}\right) .
\end{aligned}
$$

We substitute Eq. (3.8) into Eqs. (2.6) and (5.1) to eliminate $\kappa$, and then define the dimensionless quantities,

$$
\begin{aligned}
& \xi=x / x_{m} \quad \xi^{*}=x^{*} / x_{m}, \quad \theta=t \gamma m_{\infty} x_{m}^{\lambda}, \quad S=m / m_{\infty}, \\
& C=c x_{m} / m_{\infty}, \quad C^{(n)}=c^{(n)} / m_{\infty} x_{m}^{(n)}, \quad J=I / \gamma m_{\infty}^{2} x_{m}^{\lambda} .
\end{aligned}
$$

One obtains the CSD for integer values $\xi$, 


$$
\begin{aligned}
\partial C / \partial \theta= & S\left[-\xi^{\lambda} C(\xi)+(\xi-1)^{\lambda} C(\xi-1)\right] \\
& +e^{\Omega(\xi)}\left[-\xi^{\nu} C(\xi)+(\xi+1)^{\nu} C(\xi+1)\right] \\
& \times C_{\mathrm{eq}}^{(\lambda)} / C_{\mathrm{eq}}^{(\nu)}-J \delta\left(\xi-\xi^{*}\right),
\end{aligned}
$$

where $\Omega(\xi)=\alpha / \xi^{1 / 3}$ and $\xi^{*}=(\alpha / \ln S)^{3}$ in terms of $\alpha$ $=\left(4 \pi N_{A} \rho / 3 x_{m}\right)^{1 / 3} 2 \sigma \nu / R T$. In this formulation, Kelvin's relation applies to each cluster rather than to the average cluster; thus clusters larger than the critical size grow while smaller clusters dissolve. ${ }^{18}$ The mass balance for monomer, Eq. (2.6), becomes

$$
\left.d S / d \theta=-S C^{(\lambda)}+e^{\Omega} C^{(\nu)} C_{\mathrm{eq}}^{(\lambda)} / C_{\mathrm{eq}}^{(\nu)}\right)+J \xi^{*} .
$$

Here, $\Omega$ must be evaluated at the average-sized cluster, $\Omega$ $=\alpha /\left(C^{\mathrm{avg}}\right) /^{1 / 3}$. The dimensionless general moment equation is

$$
\begin{aligned}
d C^{(n)} / d \theta= & S\left[-C^{(\lambda+n)}+\sum_{j=0}^{\infty}\left(\begin{array}{c}
n \\
j
\end{array}\right) C^{(\lambda+n-j)}\right] \\
& +e^{\Omega}\left[-C^{(\nu+n)}+\sum_{j=0}^{\infty}\left(\begin{array}{c}
n \\
j
\end{array}\right)(-1)^{j} C^{(\nu+n-j)}\right] \\
& \times C_{\mathrm{eq}}^{(\lambda)} / C_{\mathrm{eq}}^{(\nu)}-J \xi^{* n} .
\end{aligned}
$$

The moment equations for $n=0,1$, and 2, Eqs. (5.5), lack closure when either $\lambda$ or $\nu$ are different from zero or unity or have noninteger values. As both $\lambda$ and $\nu$ appear even in the first moment, truncating the moment equations is not a reasonable option. Moment solutions would then not be applicable and numerical schemes have to be employed to solve the equation. An important special case is when $k_{d}$ and $k_{g}$ are independent of $x(\lambda=v=0)$; then Eq. (5.3) becomes

$$
\begin{aligned}
\dot{\partial} C(\xi) / \partial \theta= & S[-C(\xi)+C(\xi-1)] \\
& +e^{\Omega(\xi)}[-C(\xi)+C(\xi+1)]-J \delta\left(\xi-\xi^{*}\right) .
\end{aligned}
$$

The governing equation for $S(\theta)$ follows from Eq. (5.4),

$$
d S / d \theta=C^{(0)}\left(-S+e^{\Omega}\right)+J \xi^{*} .
$$

The moment equations are

$d C^{(0)} / d \theta=-J(\theta)$,

$d C^{(1)} / d \theta=C^{(0)}\left(S-e^{\Omega}\right)-J \xi^{*}$,

$d C^{(2)} / d \theta=2 C^{(1)}\left(S-e^{\Omega}\right)+C^{(0)}\left(S+e^{\Omega}\right)-J \xi^{* 2}$,

which can be solved along with Eq. (5.7). Adding Eqs. (5.7) and (5.9) gives the total mass balance,

$$
d\left(S+C^{(1)}\right) / d \theta=0
$$

The integrated mass balance, $S_{o}+C_{o}^{(1)}=S\left(\theta+C^{(1)}(\theta)\right.$, can be used to determine the approximate number of intervals needed to do the numerical analysis. Because $C^{(1)}$ $=c^{(1)} / m_{\infty} x_{m}$ is scaled by the monomer mass $x_{m}$, it is directly related to the number of monomers in the cluster. Likewise, $\xi=x / x_{m}$ is the interval in the differencedifferential equation representing the number of monomers in the cluster, and $\xi^{*}$ is the smallest number of clustered (in the nucleus). The final state is when $S_{\text {eq }}=\exp \left(\Omega_{\text {eq }}\right)$, so that the final mass (in units of number of monomers) of the larg- est cluster is $C_{\mathrm{eq}}^{(1)}=S_{o}+C_{o}^{(1)}-\exp \left(\Omega_{\mathrm{eq}}\right)$. If $C_{o}^{(1)}$ is very large $(>100)$, then $C^{(1)}$ does not change much (because $S$ is fairly close to 1 , at least not too much greater than 10). For smaller clusters $C^{(1)}$ can change quite a bit, but still only by hundreds of monomers. Note that $C^{\text {avg }}$ will always change significantly because $C^{(0)}$ approaches $1 / N_{A} m_{\infty}$. This means that we need a calculation method that is centered near $C^{\operatorname{avg}}(\theta)$ and bounded at the lower end by $\xi^{*}=(\alpha / \ln S)^{3}$, which increases as $S$ decreases. It is bounded (for all time) at the upper end by $C_{\mathrm{eq}}^{(1)}$. It is therefore possible to consider a narrow CSD with a few hundred intervals to do the numerical analysis.

Because $C(\xi, \theta)$ lies in the semi-infinite domain, it was converted to a bounded range $(0,1)$ by the mapping function, $\xi-\xi^{*}=v y /(1-y)$ with $0 \leqslant y \leqslant 1$. The grid for this mapping is fine in the range of prevalent sizes and coarse at very high and very low sizes. This ensures that $y$ varies from 0 to 1 when $\xi$ varies from $\xi^{*}$ to $\infty$. By choosing $v$ to be $C^{\text {avg }}$ $-\xi^{*}, y$ is centered at 0.5 , and the distribution is centered around $\xi=C^{\text {avg }}$, which requires fewer intervals. The initial distribution was assumed to be an exponential. The initial zeroth moment, $C_{o}^{(0)}$, is assumed to be unity.

The differential equation (5.3) was solved by RungeKutta technique with an adaptive time step. $C(\xi, \theta)$ is evaluated at each time step sequentially. Denucleation implies that the CSD is zero when $\xi \leqslant \xi^{*}$, and requires that the cluster concentration is calculated by integration of the nonzero CSD from $\xi^{*}$ to $\infty$.

The mass variable $(\xi)$ was divided into 500 intervals and the adaptive time $(\theta)$ step varied from 0.001 to 0.1 . These values ensured stability and accuracy at all values of the parameters. At every time step, the mass balance [Eq. (5.11)] is verified. Ripening is slow, with power law rather than exponential time dependence. ${ }^{1}$ Computer solutions are thus lengthy, requiring $4 \mathrm{~h}$ of CPU time on a DEC-Alpha machine for the polydispersity to reach 1.003 .

We consider a gamma initial distribution with smallest crystal mass, $x^{*}$,

$c_{o}(x)=\left[c_{o}^{(0)} / \Gamma(a) b\right]\left[\left(x-x^{*}\right) / b\right]^{a-1} \exp \left[-\left(x-x^{*}\right) / b\right]$,

which has the moments

$$
C_{o}^{(n)}=c_{o}^{(0)} \sum_{j=0}^{n}\left(\begin{array}{l}
n \\
j
\end{array}\right) x^{* n-j} j ! b^{j} \Gamma(j+a) / \Gamma(a) .
$$

Thus $c_{o}^{\mathrm{avg}}=a b+x^{*}$ and $c_{o}^{\mathrm{var}}=a b^{2}$. With $a=1$ for an initial exponential distribution, we choose the dimensionless zeroth moment, $C_{o}^{(0)}$, as unity and the supersaturation, $S(=5)$. The parameter, $\alpha$, used in computing the factor in the Kelvin equation, $\Omega\left(=\alpha / \xi^{1 / 3}\right)$ and the critical crystal size, $\xi^{*}\left(=(\alpha / \ln S)^{3}\right)$ is chosen to be equal to 5 . Figure 1 shows the evolution of the crystal size distribution for $C_{o}^{\text {avg }}$ of 75 . When $C_{o}^{\text {avg }}$ is fixed, the polydispersity [defined as $\left.C_{o}^{(2)} C_{o}^{(0)} /\left(C_{o}^{(1)}\right)^{2}\right]$ computed from Eq. (5.13) is 1.36 . The distribution evolves from an initial exponential distribution cutoff at a value of $\xi=30$, which is the initial critical cluster size, $\xi_{o}^{*}$. 


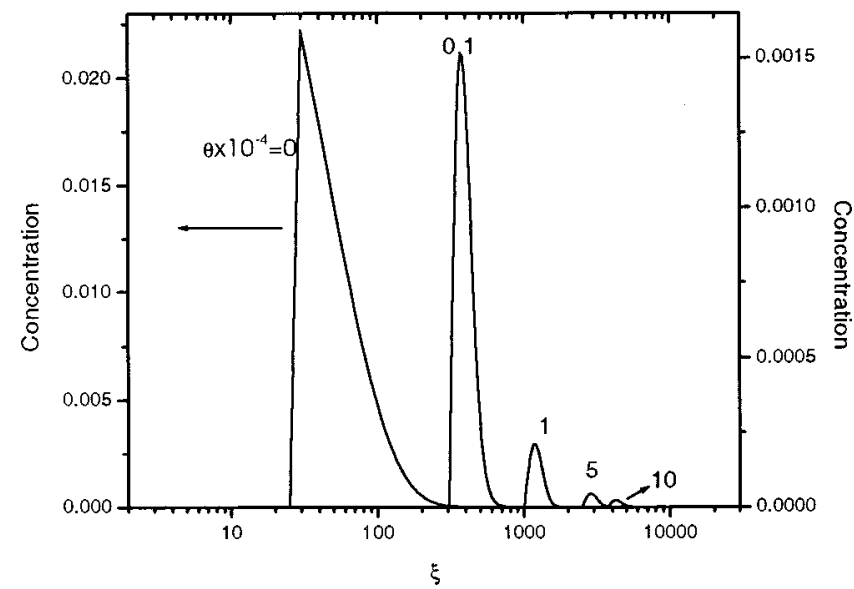

FIG. 1. Evolution of the dimensionless cluster size distribution for $\alpha=5$, $S=5, C_{o}^{(0)}=1, C_{o}^{\mathrm{avg}}=30$.

As the number of clusters declines, the CSD area and height decrease and the numerical calculation for ripening ends when the one remaining cluster is in equilibrium with the monomer phase, consistent with the mass balance based on initial total amount of monomer. Figure 2 shows the evolution of the supersaturation $S$ for four different $C_{o}^{\text {avg }}$ of 33, 50, 75, 100 and marked 1, 2, 3, 4 in the figure. Figure 3 is a $\log -\log$ plot that shows the decline of $e^{\Omega}$ with time. Figure 4 shows the time evolution of $S$ and $e^{\Omega}$ as they gradually approach each other. The $\log -\log$ plots for cluster number concentration (Fig. 5) and average cluster size (Fig. 6) show evolution to power-law behavior with time. The polydispersity $P_{D}$ (Fig. 7) evolves to a delta distribution where $P_{D}$ equals unity, independent of the initial condition, similar to the asymptotic behavior described by other investigators. ${ }^{1,8}$ For the lowest curve in Fig. 7 corresponding to $C_{o}^{\text {avg }}$ of 33 and a polydispersity of 1.008 , the initial CSD is a near-delta distribution of many clusters which increases slightly before decreasing to unity, the final CSD for a single large cluster.

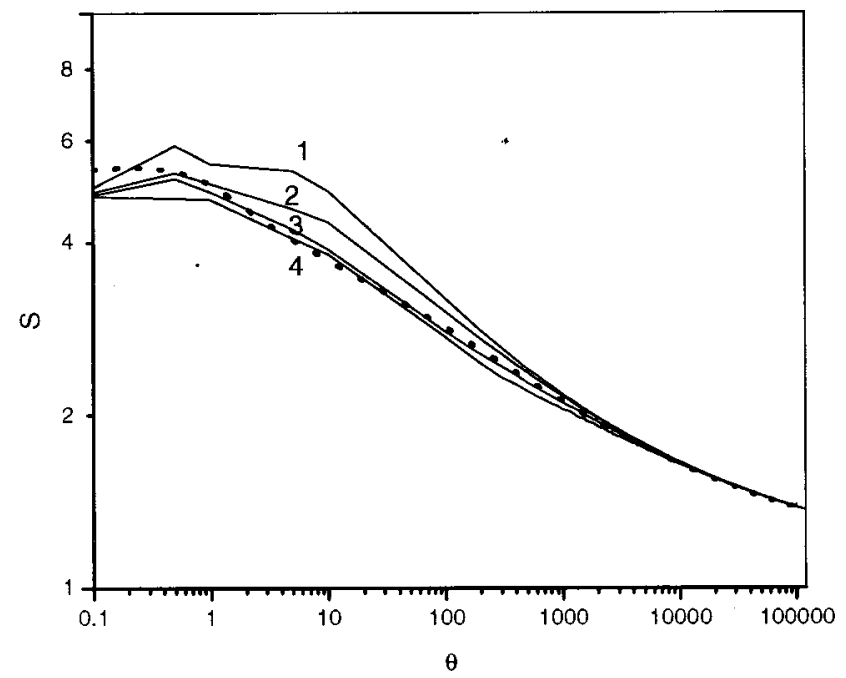

FIG. 2. Time dependence of supersaturation $S$. The dashed line is the solution of moment equations with $C_{o}^{\mathrm{avg}}=75$. The parameters used in the calculations are $\alpha=5, S=5$, and $C_{o}^{(0)}=1.1: C_{o}^{\mathrm{avg}}=33 ; 2: C_{o}^{\mathrm{avg}}=50 ; 3: C_{o}^{\mathrm{avg}}$ $=75 ; 4: C_{o}^{\mathrm{avg}}=100$.

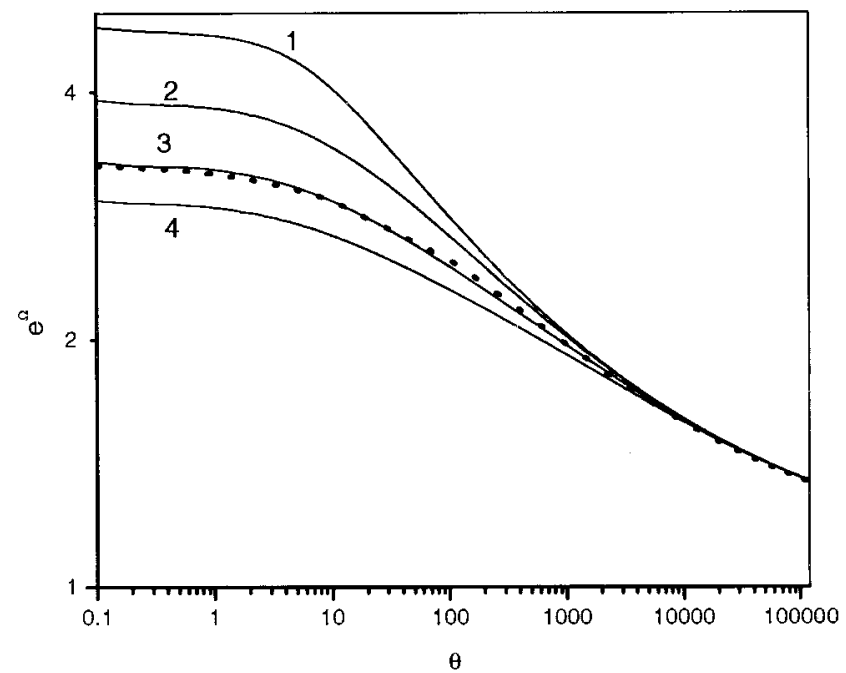

FIG. 3. $\log -\log$ plot of the variation of the Kelvin factor $e^{\Omega}$ with time. The conditions are the same as in Fig. 2.

The evolution to a single large cluster is an important feature of ripening that seems to have been avoided in some prior theories.

The ordinary differential moment equations (5.7)-(5.10) are readily solved with the NDSolve routine in Mathematica $^{\circledR}$ and are plotted in Figs. 2-7 and compared with the numerical scheme. The variation of the zeroth moment with time is given by Eq. (5.8), $d C^{(0)} / d \theta=-J(\theta)$. Because the number of crystals at any time is not known, the variation of the zeroth moment is not known a priori and a functional form for $J$ has to be assumed. The rate of denucleation, $I$ or $J$, is determined by the number of clusters of size $r^{*}$ [Eq. (3.6)], and thus requires knowledge of the CSD, which cannot be accurately constructed unless several moments are already available. In the numerical scheme, however, the variation of the zeroth moment is directly calculated because $J$ is the number of clusters removed at every time

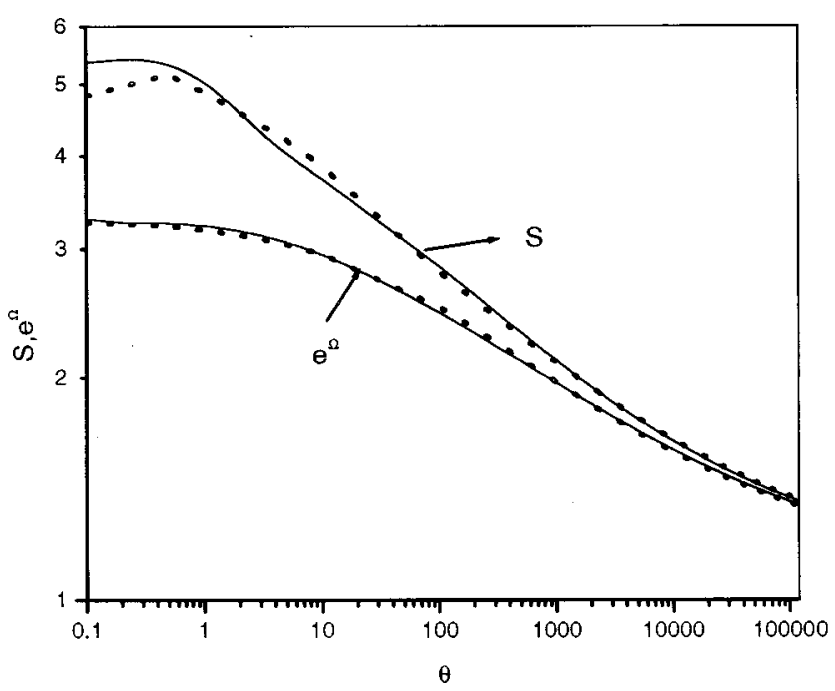

FIG. 4. Time dependence of $S$ and $e^{\Omega}$ showing the decrease of the driving force, $S-e^{\Omega}$. The parameters used in the calculations are $\alpha=5, S=5$, $C_{o}^{(0)}=1$, and $C_{o}^{\mathrm{avg}}=75$. 


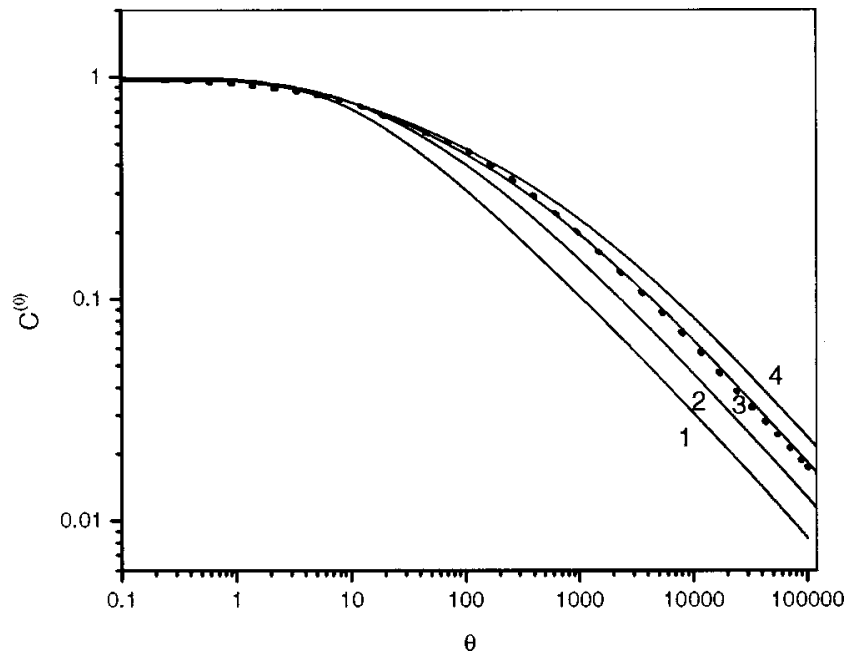

FIG. 5. Time evolution of the cluster number density showing asymptotic power-law decrease with time. The dashed line is a fit to the numerical results to allow moment calculations. See Fig. 2 for legend.

step of the computation when they reach the critical cluster size, $x^{*}$. Thus the moment solutions require the denucleation rate, $J$, as a function of $\theta$. The variation of $C^{(0)}$ with $\theta$, obtained in the numerical scheme, was fitted to a functional form, $C^{(0)}=\left[1+0.08(\theta+\epsilon)^{0.57}\right]^{-1}$. The factor, $\epsilon$, is chosen to be 0.0001 so that the differential of $C^{(0)}$ exists at $\theta=0$. As shown in Figs. 2-7, the solutions of the moment technique compare well with the solutions obtained by the numerical scheme. The moment approach, however, has the severe disadvantage that it does not allow a straightforward estimation of the denucleation rate, $J$, because one needs the actual CSD to know the number of clusters at any time $t$. In the numerical solution of the population balance equation, $J$ is the number of clusters removed at every time step of the computation when they reach the critical cluster size, $x^{*}$.

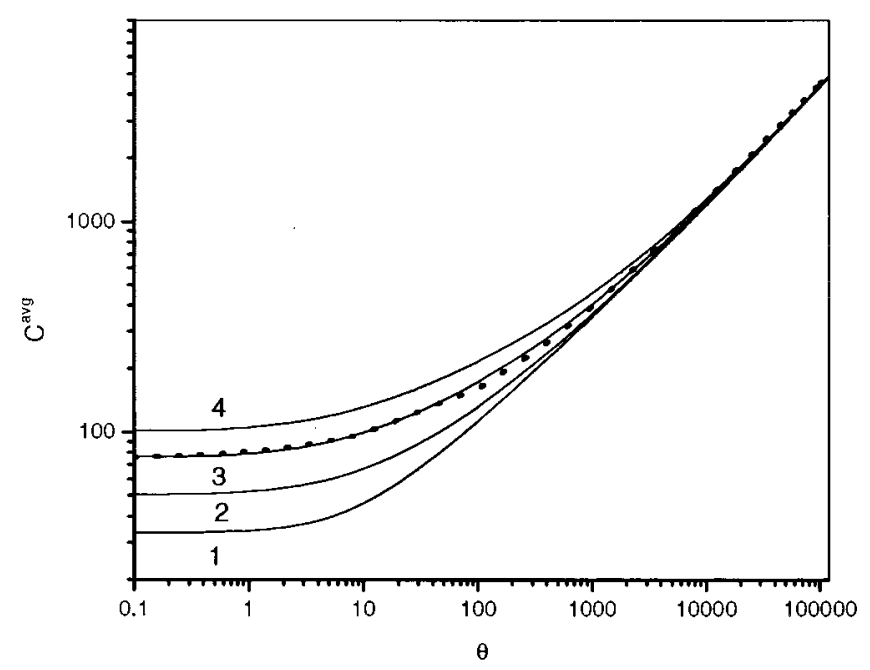

FIG. 6. Time evolution of the average cluster mass showing asymptotic power-law increase with time. Other conditions are same as Fig. 2.

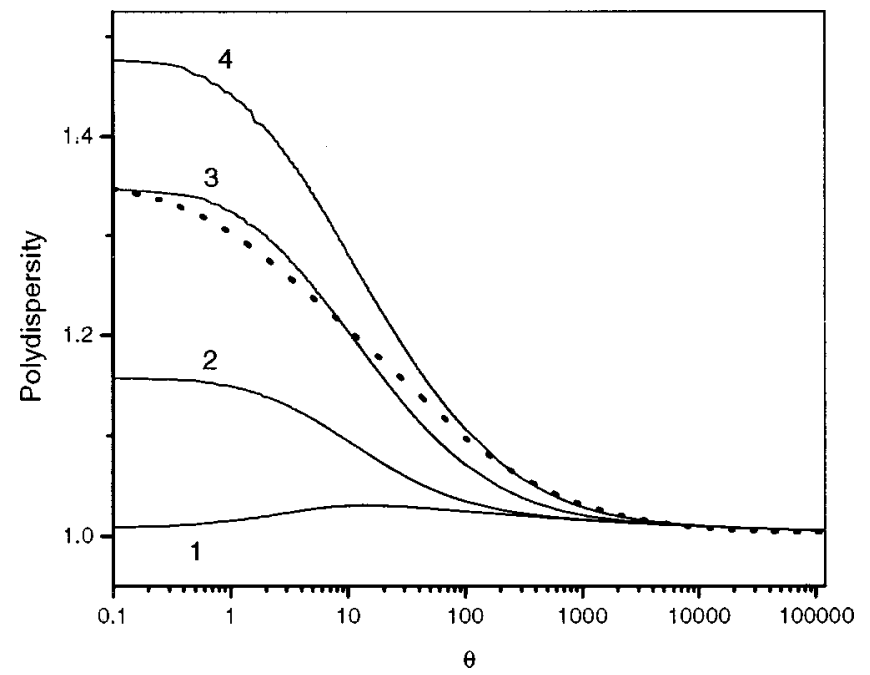

FIG. 7. Polydispersity as a function of time. Other conditions are same as in Fig. 2.

\section{CONCLUSION}

Because ripening occurs for a distribution of particle sizes, the governing equations must describe how such a distribution evolves with time. The thermodynamic effect of size on solubility is the main driving force for this evolution. As smaller, more soluble clusters dissolve and vanish, they give up their mass to larger clusters that are growing. The present distribution kinetics theory emphasizes the importance of particle loss in Ostwald ripening. Only when such denucleation is accounted for can the final state of one large particle be realized. The population balance equations of this work are based on mass conservation, similar to the distribution kinetics of chain polymerization. Numerical solutions show the growth and broadening of the CSD in agreement with moment solutions for the average and variance. As larger clusters grow while smaller clusters dissolve and eventually disappear, the average cluster mass steadily increases. Finally, one cluster remains in equilibrium with the monomer solution. By numerical integration of a discrete PBE, derived as a special case of the continuous PBE and represented as a difference-differential equation, the asymptotic nature of the CSD at long time is realized. The moment solutions also match the results obtained from numerical computations. However, the denucleation rate has to be determined from the numerical computation for use in the moment equations.

\section{ACKNOWLEDGMENT}

This work was supported in part by NSF Grant No. CTS-9810194.

\section{APPENDIX}

A moment theory for ripening in terms of the cluster radius ${ }^{1,7,8,12,13,18}$ (rather than mass, $x$ ) can be fashioned from Eqs. (4.3)-(4.5). The conventional procedure is to consider the particle distribution defined by $p(r, t) d r$, which at time $t$ is the number of clusters having radii in $(r, r+d r)$. The moments are defined by 


$$
p^{(n)}(t)=\int_{o}^{\infty} p(r, t) r^{n} d r .
$$

Then the zeroth moment, $p^{(0)}(t)$, is the number density of particles, the first moment is related to the average radius as $p^{\text {avg }}(t)=p^{(1)}(t) / p^{(0)}(t)$, the second moment, $p^{(2)}(t)$, is proportional to the total particle surface area per unit volume, and the third moment, $p^{(3)}(t)$, is proportional to the total particle volume (or mass) per unit vessel volume. The PBE is

$$
\partial p(r, t) / \partial t+\partial\left(r^{*} p\right) / \partial r=-I(t) \delta(r-*),
$$

where $I(t)$ is the time-dependent denucleation rate of clusters of radius $r^{*}$. The growth (or dissolution) rate is given by

$$
r^{\bullet}=k\left[m(t)-m_{\mathrm{eq}}\right],
$$

where

$$
m_{\text {eq }}=m_{\infty} \exp (\Omega)
$$

causes larger clusters to grow faster than smaller clusters. This form of the growth term follows the usual expression, ${ }^{1,8}$ except that we do not linearize the exponential. We assume here that the radius in $\Omega$ is the average particle radius, thus

$$
\Omega=\alpha^{\prime \prime} / p^{\text {avg }}(t),
$$

where $\alpha^{\prime \prime}=2 \sigma \nu / R T$.

The moment equations for the PBE (A2) can be found by integration of the second term by parts,

$$
d p^{(n)} / d t-n k\left[m(t)-m_{\infty} \exp (\Omega)\right] p^{(n-1)}=-I(t) r^{* n} .
$$

A mass balance on the solubilized monomer and cluster mass corresponds to Eq. (2.10),

$$
d m / d t=-\left(4 \pi \rho / 3 x_{m}\right) d p^{(3)} / d t
$$

so that a gain in cluster mass concentration by growth is balanced by a loss of solute of $M W \cdot x_{m}$. The moment equations [Eq. (A6) for $n=0,1,2,3]$ are as follows:

$$
\begin{aligned}
& d p^{(0)} / d t=-I(t), \\
& d p^{(1)} / d t=k\left[m(t)-m_{\infty} \exp (\Omega)\right]^{(0)}-I(t) r^{*},
\end{aligned}
$$

$$
\begin{aligned}
& d p^{(2)} / d t=2 k\left[m(t)-m_{\infty} \exp (\Omega)\right] p^{(1)}-I(t) r^{* 2}, \\
& d p^{(3)} / d t=3 k\left[m(t)-m_{\infty} \exp (\Omega)\right] p^{(2)}-I(t) r^{* 3} .
\end{aligned}
$$

Moments up through third order are required to achieve closure [by Eq. (A7)]. Differential Eqs. (A9)-(A13) are easily solved with Eq. (A5) depending on time through $p^{\text {avg }}(t)$. Equation (A9) shows that the number of clusters decreases with time. At equilibrium we have $I=0$ and $d p^{(n)} / d t=0$, which yields

$$
m_{\mathrm{eq}}^{(0)}=m_{o}^{(0)}-\left(4 \pi \rho / 3 x_{m}\right)\left[p_{\mathrm{eq}}^{(3)}-p_{o}^{(3)}\right]
$$

and satisfies the mass balance. As the number of particles becomes very small, the average cluster mass increases to the one remaining large particle. Unfortunately, this moment theory suffers the disadvantage that there is no straightforward way to choose an expression for $I(t)$. A moment theory based on $c(x, t)$ has the same difficulty.

${ }^{1}$ J. A. Marqusee and J. Ross, J. Chem. Phys. 79, 373 (1983).

${ }^{2}$ B. J. McCoy, J. Colloid Interface Sci. 228, 64 (2000).

${ }^{3}$ B. J. McCoy, J. Colloid Interface Sci. 240, 139 (2001).

${ }^{4}$ A. E. Nielsen, Kinetics of Precipitation (Pergamon, Oxford, 1964).

${ }^{5} \mathrm{O}$. Sohnel and J. Garside, Precipitation-Basic Principles and Industrial Applications (Butterworth-Heinemann, Ltd., Oxford, 1992).

${ }^{6}$ A. W. Adamson and A. P. Gast, Physical Chemistry of Surfaces, 6th ed. (Wiley-Interscience, New York, 1997).

${ }^{7}$ A. Bhakta and E. Ruckenstein, J. Chem. Phys. 103, 7120 (1995).

${ }^{8}$ G. Venzl, Ber. Bunsenges. Phys. Chem. 87, 318 (1983).

${ }^{9}$ J. W. Morse and W. H. Casey, Am. J. Sci. 288, 537 (1988).

${ }^{10}$ G. Venzl, Phys. Rev. A 31, 3431 (1985).

${ }^{11}$ A. C. Lasaga, Kinetic Theory in the Earth Sciences (Princeton University Press, New Jersey, 1998).

${ }^{12}$ I. M. Lifshitz and J. Slyozhov, J. Phys. Chem. Solids 19, 35 (1961).

${ }^{13}$ C. Wagner, Z. Elektrochem. 65, 243 (1961).

${ }^{14}$ Y. Kodera and B. J. McCoy, AIChE J. 43, 3205 (1997).

${ }^{15}$ R. L. Cotterman, R. Bender, and J. M. Prausnitz, Ind. Eng. Chem. Process Des. Dev. 24, 194 (1985).

${ }^{16}$ B. J. McCoy and G. Madras, Chem. Eng. Sci. 56, 2831 (2001).

${ }^{17}$ N. G. van Kampen, Stochastic Processes in Physics and Chemistry (Elsevier Science, North-Holland, 1992).

${ }^{18}$ W. J. Dunning, "Ripening and ageing processes in precipitates," in Particle Growth in Suspensions, edited by A. L. Smith (Academic, New York, 1973). 